\title{
Commentary \\ The value of a risk model for early-onset candidemia
}

\section{Christian Sandrock and Javeed Siddiqui}

\begin{abstract}
Division of Pulmonary and Critical Care, Division of Infectious Diseases, University of California Davis School of Medicine, 4150 V Street \#3400, Sacramento, CA 95817, USA
\end{abstract}

Corresponding author: Christian Sandrock, cesandrock@ucdavis.edu

Published: 16 November 2009

This article is online at http://ccforum.com/content/13/6/1005

(c) 2009 BioMed Central Ltd

See related research by Shorr et al., http://ccforum.com/content/13/5/R156
Critical Care 2009, 13:1005 (doi:10.1186/cc8127)

\begin{abstract}
Bloodstream infections from Candida species are associated with an increased length of stay, increased hospital costs, and higher mortality when compared with bacterial bloodstream infections. Delayed or inappropriate therapy in candidemia leads to increased mortality, thus early recognition becomes paramount. With biomarkers showing promise, blood cultures still remain the gold standard but require 24 to 72 hours for growth. The reliance on epidemiologic risk factors for the initiation of empiric antifungal therapy therefore provides the best method for early appropriate therapy. Shorr and colleagues have devised a risk score to identify patients with early-onset candidemia as defined by positive blood cultures within 2 days of admission, thus allowing for the initiation of early appropriate antifungal therapy.
\end{abstract}

In a previous edition of Critical Care, Shorr and colleagues developed a simple weight risk score for identifying patients with candidemia upon hospital admission [1]. Using recursive partitioning, they determined the best discriminators of Candida bloodstream infections in patients upon hospitalization (identified as a positive blood culture 1 day prior to or 2 days after admission) by retrospectively reviewing the CareFusion Outcomes Research Database, comprising 64,109 bloodstream infection cases admitted to 176 acute care hospitals from 2000 to 2005 . Three sets of models were applied (equal weight, unequal weight, and full weight with additional variables) for sensitivity analysis. The risk score was then validated using the 2006/2007 year cohort for a total of 24,685 bloodstream infections.

The rate of candidemia was $1.2 \%$ of all bloodstream infections for the 5-year derivation cohort, and was $1.3 \%$ for the validation cohort. The rate was increased to $2.3 \%$ and $3.1 \%$, respectively, for those patients with mechanical ventilation. Baseline characteristics were largely similar between both cohorts, and univariate analysis determined that the following risk factors are associated with candidemia: age $\leq 64$ years; cachexia; deranged albumin, arterial $\mathrm{pH}$, and electrolytes; temperature $\leq 98^{\circ} \mathrm{C}$ or fever; altered mental status; previous hospitalization within 30 days; admission from another healthcare facility; and mechanical ventilation. Recursive partitioning revealed that the six best discriminators are age $<64$ years, temperature $<98^{\circ} \mathrm{C}$, cachexia, previous hospitalization, admission from another healthcare facility, and mechanical ventilation.

In the derivation cohort, those patients with one risk factor had a rate of candidemia of $0.4 \%$ while those with all six risk factors had a rate of $27.3 \%$. In the validation cohort, the rates of candidemia were similar through the risk factor stratification groups. The area under the receiver operating curve for the risk score was 0.70 for the derivation cohort and was 0.71 for the validation cohort. With the model involving six risk factors, the area under the receiver operating curve was similar in both cohorts. Finally, the area under the receiver operating curve for the model with 16 risk factors was associated with a slightly higher discrimination in both cohorts; but on recalibration with the validation cohort, seven risk variables were deemed poor discriminators - thus suggesting that additional factors did not improve the risk model during validation.

Even though the rates of candidemia bloodstream infections on admission are low, the authors conclude that with certain epidemiologic risk factors the rates increase from 1 in 500 to 1 in 4 bloodstream infection admissions. A validated risk model based on these six discriminators (age <64 years, temperature $<98^{\circ} \mathrm{C}$, cachexia, previous hospitalization, admission from another healthcare facility, and mechanical ventilation) may therefore provide early detection and subsequent early appropriate treatment of these high-risk patients, potentially improving outcome.

Candidemia is the fourth most common bloodstream isolate in hospitalized patients and accounts for an increased length of stay and significant morbidity and mortality, ranging from 25 to $58 \%$ [2-4]. Many of these data have been evaluated in 
hospitalized patients, however, where the epidemiologic data and risk factor analysis have been more developed. Other predictive scores have included candida colonization, parenteral nutrition, and antibacterial therapy - these factors are common in hospitalized intensive care unit patients, which constitute most cases of candidemia $[5,6]$.

Early-onset candidemia, as defined by a positive blood culture within 2 days of admission, is a less described entity. Shorr and colleagues, in another publication, have recently outlined the burden of early-onset candidemia, with a longer length of stay, higher crude mortality, and higher hospitalization cost when compared with bacterial bloodstream infections [7]. While the rates of candidemia remain low (1.3\% of all bacteremia cases), certain high-risk patients have much higher rates approaching $27 \%$ as outlined in this study. Risk stratification by application of the validated risk model can have a profound impact on early therapy and intervention in these cases.

The role of early appropriate therapy has become important as inappropriate or delayed therapy leads to higher mortality [8-10]. In bacterial bloodstream infections and pneumonia, early identification of those at risk for multidrug-resistant organisms can lead to early appropriate therapy, and thus to a lower mortality [9]. In candidemia, studies have show that delayed therapy can lead to a higher mortality in hospitalized patients with late candidemia [11]. Early identification of these patients therefore becomes paramount. Since earlyonset candidemia is an unusual presentation on hospitalization, a high potential for delayed therapy exists - even in the high-risk groups. While diagnostic biomarkers ( $\beta$-D-glucan) have promise, blood cultures still remain the gold standard for diagnosis but take 24 to 72 hours for growth.

Risk analysis models or scores have been used in the past for prophylaxis or empiric therapy for candidemia $[5,6]$. These scores have been in hospitalized patient populations, however, largely in the intensive care unit where candidemia is more prevalent. A risk factor model has not been used before in early-onset candidemia. The use of recursive partitioning for development of risk determinants has been used in prior bacterial bloodstream infections in pediatric patients, but this is its first use in candidemia of any kind [12]. The development of a risk model as described by Shorr and colleagues therefore becomes a useful tool in determining the highest risk individuals for early-onset candidemia, thereby allowing early appropriate empiric therapy for this subset of patients.

As we have evolved over the past decade to recognize and treat high-risk individuals for multidrug-resistant pneumonia (for example, healthcare-associated pneumonia), Shorr and colleagues' risk model allows for that initial discrimination of the high-risk groups. For the next step we need to evaluate its impact in prospective studies, particularly evaluating various risk models and the impact on early appropriate therapy, morbidity, mortality, and Candida resistance patterns. A risk model for early-onset candidemia, however, is a starting point.

\section{Competing interests}

The author declares that they have received funding from Estellas and Pfizer.

\section{References}

1. Shorr AF, Tabak YP, Johannes RS, Sun X, Spalding J, Kollef MH: Candidemia on presentation to the hospital: development and validation of a risk score. Crit Care 2009, 13:R156.

2. Miller PJ, Wenzel RP: Etiologic orangisms as independent predictors of death and morbidity associated with blood stream infections. J Infect Dis 1987, 156:471-477.

3. Macphail GL, Taylor GD, Buchanan-Chell M, Ross C, Wilson S, Kureishi A: Epidemiology, treatment and outcome of candidemia: a five-year review at three Canadian hospitals. Mycoses 2002, 45:141-145.

4. Kung HC, Wang JL, Chang SC, Wang JT, Sun HY, Hsueh PR, Chen YC: Community-onset candidemia at a university hospital, 1995-2005. J Microbiol Immunol Infect 2007, 40:355-363.

5. León C, Ruiz-Santana S, Saavedra P, Galván B, Blanco A, Castro C, Balasini C, Utande-Vázquez A, González de Molina FJ, BlascoNavalproto MA, López MJ, Charles PE, Martín E, Hernández-Viera MA: Usefulness of the 'Candida score' for discriminating between Candida colonization and invasive candidiasis in non-neutropenic critically ill patients: a prospective multicenter study. Crit Care Med 2009, 37:1624-1633.

6. León C, Ruiz-Santana S, Saavedra P, Almirante B, Nolla-Salas J, Alvarez-Lerma F, Garnacho-Montero J, León MA: A bedside scoring system ('Candida score') for early antifungal treatment in nonneutropenic critically ill patients with Candida colonization. Crit Care Med 2006, 34:730-737.

7. Shorr AF, Gupta V, Sun X, Johannes RS, Spalding J, Tabak YP: Burden of early-onset candidemia: analysis of culture-positive bloodstream infections from a large U.S. database. Crit Care Med 2009, 37:2519-2526.

8. Craven DE, Palladino R, McQuillen DP: Healthcare-associated pneumonia in adults: management principles to improve outcomes. Infect Dis Clin North Am 2004, 18:939-962.

9. Ibrahim EH, Sherman G, Ward S, Fraser VJ, Kollef MH: The influence of inadequate antimicrobial treatment of bloodstream infections on patient outcomes in the ICU setting. Chest 2000, 118:146-155.

10. Kollef MH, Sherman G, Ward S, Fraser VJ: Inadequate antimicrobial treatment of infections: a risk factor for hospital mortality among critically ill patients. Chest 1999, 115:462-474.

11. Garey KW, Rege M, Pai MP, Mingo DE, Suda KJ, Turpin RS, Bearden DT: Time to initiation of fluconazole therapy impacts mortality in patients with candidemia: a multi-institutional study. Clin Infect Dis 2006, 43:25-31.

12. Bachur RG, Harper MB: Predictive model for serious bacterial infections among infants younger than 3 months of age. Pediatrics 2001, 108:311-316. 